Exercise Physiology

\title{
Physically active routine during COVID-19 pandemic: do not fail to comply with the recommendations for cardiovascular health
}

\author{
Maycon Junior Ferreira ${ }^{1}$ (D) , Maria Cláudia Irigoyen ${ }^{2}$ (D) , Kátia De Angelis ${ }^{1,3}$ (D) \\ ${ }^{1}$ Universidade Federal de São Paulo, Departamento de Fisiologia, São Paulo, SP, Brasil; \\ ${ }^{2}$ Universidade de São Paulo, Faculdade de Medicina, Instituto do Coração (InCor), São Paulo, SP, \\ Brasil, ${ }^{3}$ Universidade Nove de Julho, São Paulo, SP, Brasil.
}

Associate Editor: Angelina Zanesco. UNESP/Rio Claro, SP, Brasil.

Keywords: physical activity; COVID-19; sedentarism; cardiovascular risk; prevention.

\section{COVID-19 \& cardiovascular disease: impact of sedentarism}

The rapidly-evolving outbreak of the new Coronavirus (SARSCoV-2) worldwide, combined with its potential severity, led the World Health Organization (WHO) to characterize it as a pandemic, conducting several countries to adopt containment strategies to curb the pandemic, such as quarantine and stringent social distancing. In addition to essential preventive measures, public policies aimed at reducing cardiovascular $(\mathrm{CV})$ risk factors will be required during and after this infectious scenario. In this context, cardiovascular prevention strategies will have to be strengthened, since they are crucial for the reduction of medium and long-term risk of $\mathrm{CV}$ mortality.

The health-damaging potential of the sedentary behavior pattern emerging from social confinement has posed another hurdle in the fight against the SARS-CoV-2, and its impact on the severity of the disease should not be neglected. It is widely known that sedentary behavior is associated with an increased risk of all-cause $\mathrm{CV}$ and cancer mortality ${ }^{1}$. Since the beginning of the pandemic, a high prevalence of physical inactivity-associated comorbidities in COVID-192-4, such as hypertension (HTN) and diabetes mellitus (DM) has been identified. Importantly, more severe forms of COVID-19 have been found in patients with these preexisting $\mathrm{CV}$ risk factors ${ }^{5}$, suggesting that such factors play a strong role in both individuals susceptible to infection and patients infected with SARS-CoV-2.

The psychological distress caused by quarantine has been widely reported ${ }^{6}$ and chronic stress may diminish the motivation to engage in any physical activity ${ }^{7}$. In addition, social isolation associated with feelings of loneliness leads to elevated CV risk $^{8}$, and the risk of coronary heart disease and stroke due to a poor social relationship increase 32 e $29 \%$, respectively 9 . Individuals often resort to excess caloric intake for stress relief, favoring weight gain, an important trigger for the development of comorbidities such as obesity and metabolic syndrome. Lower energy expenditure due to actual reduced mobility may become a health issue and should be addressed as such. The SARS-CoV-2 pandemic exacerbated the vicious circle of stress-fatty junk food overconsumption that had been in place for decades, further worsening an obesogenic scenario by the abrupt reduction in physical activity levels.

\section{Physically active lifestyle positively impacts cardiovascular health and COVID-19 complications}

Both the practice of physical activity (understood as any motor activity that results in energy expenditure above resting levels) and exercise (systematized practice, carefully designed and prescribed, considering training variables and aiming at specific objectives) are of fundamental importance for this period of social distancing, particularly for the individuals with CV risk factors, which need closer and longer monitoring, due to their greater susceptibility to COVID-19 infection and complications. The current WHO guideline recommended at least 150-300 minutes of moderate-intensity throughout the week for adults and at least an average of 60 minutes per day of moderate- to vigorous-intensity across the week for children and adolescents ${ }^{10}$. However, even at levels lower than recommendations, physical activity may have a protective effect on COVID- $19^{11}$. Therefore, any body movement, according to one's routine, could play a beneficial role during and after the pandemic (Figure 1).

Many countries have already relaxed quarantine and social distance measures. However, both SARS-CoV-2 infection and some degree of social distance are predicted to last much longer. Given the uncertainties surrounding COVID-19 treatment and vaccine in the near future, addressing sedentary behavior and the associated-CV risks will remain a key health demand, requiring the immediate adoption of effective strategies to promote a physically active lifestyle during the pandemic. In recent decades, there has been a significant drop in CV diseases in several countries, fueled by the science-informed fostering of a healthy active lifestyle ${ }^{12}$. The emergence of COVID-19 
required confinement measures, however, may not only reverse this achievement but lead to a huge step back, as sedentary behavior in the context of the pandemic may further increase the risk of CV mortality. Thus, it is critical to encourage individuals to engage in physical activity, within their physical limitations, maintaining the use of prescribed medications, following medical recommendations, and seeking medical advice in the presence of any signs of discomfort when exercising.

Infected patients by SARS-CoV-2 requiring hospitalization may experience a dramatic reduction in cardiorespiratory fitness. Given the significant potential of cardiorespiratory fitness in abolishing $\mathrm{CV}$ risk related to sedentarism ${ }^{13}$, exercise-based telerehabilitation programs are highly desirable for this population and may favor a less traumatic and quicker recovery.

\section{Final Remarks}

The impact of COVID-19 could strongly affect economic, public, and health systems ${ }^{14}$. The maintenance of a physically active lifestyle as a preventive health strategy plays a key role in the fight against potential deleterious outcomes of COVID-19 and CV diseases. In face of the COVID-19 pandemic, for which treatment and vaccines are still in early development, the coming years will be indeed challenging. The negative behavioral changes induced by the virus should also be a reminder that sedentarism-associated $\mathrm{CV}$ diseases also cross borders and will not benefit from vaccines. However, the remedy may simply lie in a heightened awareness of the importance of physical exercise and the sustained engagement of health authorities and the population.

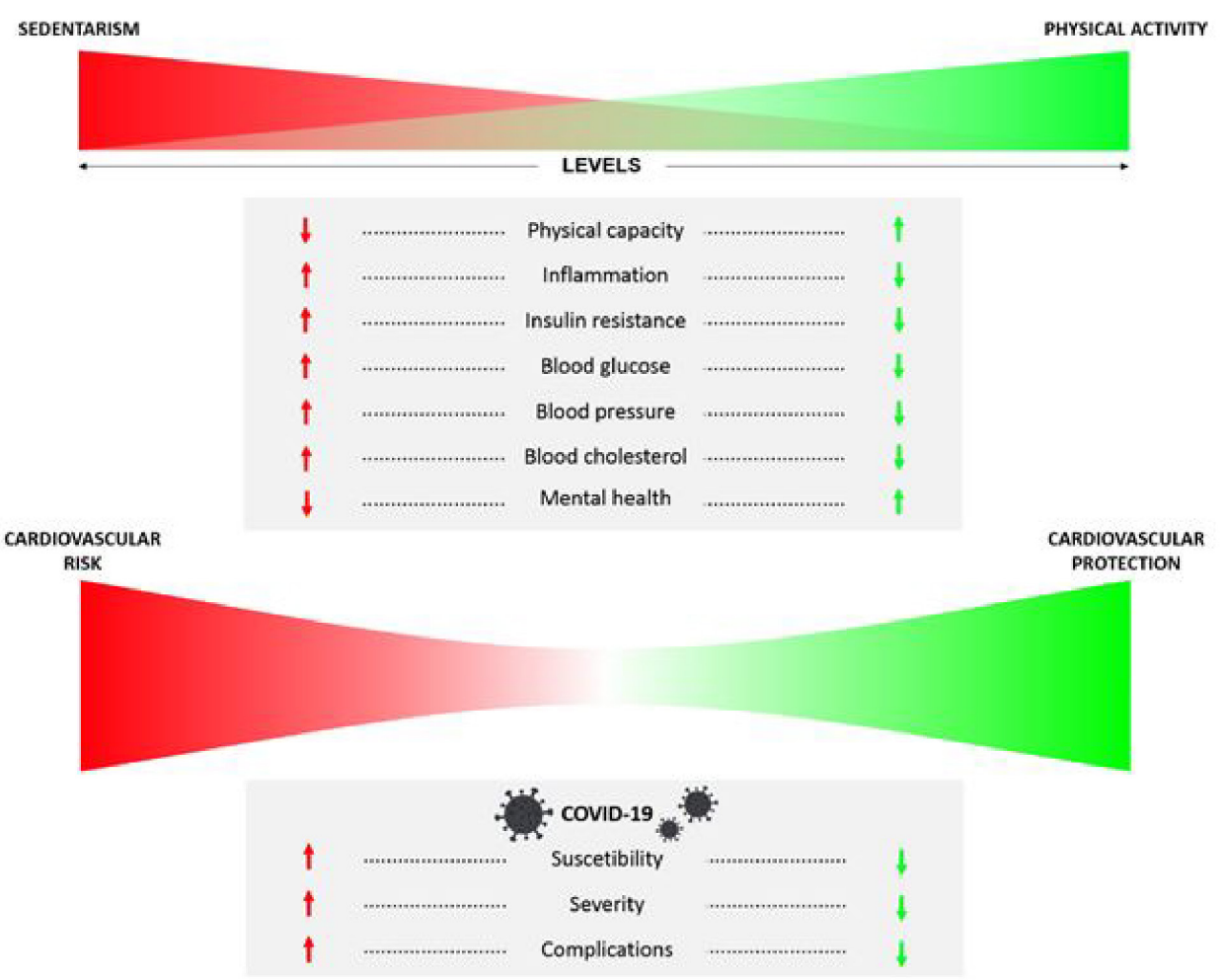

Figure 1 - The potential effects of sedentarism and physical activity during long-term on health parameters, and possible consequences of COVID-19 disease. High levels of sedentarism and low physical activity during long periods can trigger changes in physical, metabolic, cardiovascular and inflammation parameters, resulting in an elevated cardiovascular risk in the future, and predisposing individuals to a higher risk of SARS-CoV-2-associated disease, whereas the reduction in sedentarism and increased physical activity levels can to meliorating these components of health, reducing the cardiovascular risk and potentially improvement the protection against the COVID-19.

\section{References}

1. Patterson R, McNamara E, Tainio M, de Sá TH, Smith AD, Sharp SJ, et al. Sedentary behaviour and risk of all-cause, cardiovascular and cancer mortality, and incident type 2 diabetes: a systematic review and dose response meta-analysis. Eur J Epidemiol. 2018;33(9):811-29.

2. Zhou F, Yu T, Du R, Fan G, Liu Y, Liu Z, et al. Clinical course and risk factors for mortality of adult inpatients with COVID-19 
in Wuhan, China: a retrospective cohort study. Lancet. 2020;395(10229):1054-62.

3. Wu C, Chen X, Cai Y, Xia J, Zhou X, Xu S, et al. Risk factors associated with acute respiratory distress syndrome and death in patients with coronavirus disease 2019 pneumonia in Wuhan, China. JAMA Intern Med. 2020;180(7):1-11.

4. Tian J, Yuan X, Xiao J, Zhong Q, Yang C, Liu B, et al. Clinical characteristics and risk factors associated with COVID-19 disease severity in patients with cancer in Wuhan, China: a multicentre, retrospective, cohort study. Lancet Oncol. 2020;21(7):893-903.

5. Krittanawong C, Virk HUH, Narasimhan B, Wang Z, Narasimhan H, Zhang HJ, et al. Coronavirus disease 2019 (COVID-19) and cardiovascular risk: A meta-analysis. Prog Cardiovasc Dis. 2020;63(4):527-8.

6. Brooks SK, Webster RK, Smith LE, Woodland L, Wessely S, Greenberg N, et al. The psychological impact of quarantine and how to reduce it: rapid review of the evidence. Lancet. 2020;395(10227):912-20.

7. Stults-Kolehmainen MA, Sinha R. The effects of stress on physical activity and exercise. Sport Med. 2014;44(1):81-121.

8. Xia N, Li H. Loneliness, social isolation, and cardiovascular health. Antioxidants Redox Signal. 2018;28(9):837-51.

9. Valtorta NK, Kanaan M, Gilbody S, Ronzi S, Hanratty B. Loneliness and social isolation as risk factors for coronary heart disease and stroke: systematic review and meta-analysis of longitudinal observational studies. Heart. 2016;102(13):1009-16.

10. World Health Organization. WHO Guidelines on Physical Activity and Sedentary Behaviour. Geneva; 2020. Available from: https:// www.who.int/publications/i/item/9789240015128 [Accessed 5th February 2021].

11. Hamer M, Kivimäki M, Gale CR, David Batty G. Lifestyle risk factors, inflammatory mechanisms, and COVID-19 hospitalization: A community-based cohort study of 387,109 adults in UK. Brain Behav Immun. 2020;87:184-7.

12. Zou Z, Cini K, Dong B, Ma Y, Ma J, Burgner DP, et al. Time trends in cardiovascular disease mortality across the BRICS: an age-period-cohort analysis of key nations with emerging economies using the global burden of disease study 2017. Circulation. 2020;141(10):790-9.

13. Nauman J, Stensvold D, Coombes JS, WislØff U. Cardiorespiratory fitness, sedentary time, and cardiovascular risk factor clustering. Med Sci Sports Exerc. 2016;48(4):625-32.

14. Carter P, Anderson M, Mossialos E. Health system, public health, and economic implications of managing COVID-19 from a cardiovascular perspective. Eur Heart J. 2020;41(27):2516-8.

\section{Corresponding author}

Kátia De Angelis. Federal University of São Paulo (UNIFESP), Department of Physiology ( $5^{\circ}$ floor), Botucatu Street, 862, Vila Clementino, 04023-062, São Paulo, SP, Brazil. Telephone: +55 (11) 5573-9525.

Email: prof.kangelis@yahoo.com.br

Manuscript received on November 16, 2020

Manuscript accepted on November 17, 2020

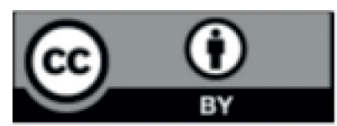

Motriz. The Journal of Physical Education. UNESP. Rio Claro, SP, Brazil - eISSN: 1980-6574 - under a license Creative Commons - Version 4.0 